\title{
UNA NUEVA PROPUESTA DIDÁCTICA PARA LA ENSEÑANZA DE LA RELATIVIDAD EN EL BACHILLERATO
}

\author{
ALEMAÑ BERENGUER, R.A ${ }^{1}$. y PÉREZ SELLES, J.F. ${ }^{2}$ \\ ${ }^{1}$ IES núm. 2. El Campello. Alicante \\ ${ }^{2}$ IES núm. 4. Benidorm. Alicante
}

\section{SUMMARY}

The great importance that has been given to teaching the theory of Relativity in intermediate levels by the new plans of secondary education, is often discouraged by the erroneous treatment of all these topics in the usual didactic formulations. So, in this work we dare to suggest a new point of view which is more accurately attached to the real and original spirit of the genuine Relativity. Our proposal lies on recovering in teaching the ideas of space-time and geometric representations introduced by Minkowski.

\section{INTRODUCCIÓN}

Una de las virtudes que ha mostrado la reforma educativa en el ámbito de la física ha sido la de modernizar el enfoque de los temarios que hasta el momento se venían impartiendo en el nivel de la educación preuniversitaria (MEC, 1992). Semejante puesta al día, tan necesaria como era, comportaba entre otros aspectos, el conceder un lugar destacado a temas de física moderna, como son la física relativista, la física cuántica y la física nuclear y de partículas, las cuales ya se encontraban en la mayoría de los textos de COU, si bien no se incluían entre los temas de selectividad exigidos en muchas comunidades autónomas. Las unidades didácticas sugeridas por las autoridades educativas de diversos territorios sobre el tema de la relatividad diferían muy poco entre sí. Por ese motivo era de esperar que los errores o deficiencias detectadas en algunas de tales unidades didácticas se repitiesen en las otras.

Y así ha sido. Consultando diversos materiales curriculares (Solbes, 1993; Calvo y Seguí, 1996), hemos podido constatar los errores conceptuales que, en lo referente al tema de relatividad, arrastran todos ellos. Ése ha sido uno de los principales motivos de la elaboración de la presente propuesta que pretende llenar los vacíos detectados y solventar los malentendidos que todavía persisten en la docencia de esta apasionante rama de la física. La base de nuestra alternativa consiste en recuperar, en la medida de lo posible, el planteamiento geométrico en un espacio-tiempo de cuatro dimensiones (Penrose, 1991) que recibió la relatividad especial a partir de 1907 de mano de Minkowski (Sazànov, 1988). Este formalismo -tan novedoso hoy como entonces para la mayoría de los docentes de secundaria y para algunos de sus asesores didácticos- fue recibido inicialmente con desconfianza por el propio Einstein (Sánchez Ron, 1983), hasta que, en busca de la relatividad general, se adhirió por fin a él hacia 1911 (Schwinger, 1995).

Es importante hacer dos salvedades previas al desarrollo de la propuesta que se expondrá a continuación. Nuestro trabajo pretende ser un refinamiento y mejora de las unidades didácticas utilizadas hasta el presente sin albergar el ánimo de descalificarlas por completo. De hecho, los primeros apartados, dedicados usualmente a 
la introducción de las carencias en la física clásica que condujeron al desarrollo de la física moderna, resultan del todo aceptables a nuestro juicio. Así pues, hemos preferido no insistir en dichos aspectos, y ésa es la razón del sucinto tratamiento que en nuestra propuesta alternativa se concede a la búsqueda de un referencial en reposo absoluto o al experimento de Michelson, por ejemplo. En esos casos deberá entenderse que el tratamiento tradicional se considera válido y su repetición resultaría superflua.

En segundo lugar, nuestra propuesta pretende no ser más que lo que su propio nombre indica: un proyecto sugerido, un documento de base para un trabajo posterior de elaboración. Por ello, ni aspiramos a poseer la receta infalible para la docencia adecuada de estos contenidos ni podemos aportar abundantes experiencias de aula. Precisamente el hecho de que se trate de un planteamiento novedoso nos imposibilita para exhibir esos dos méritos. Pero no por ello dejamos de estar convencidos de que la única manera de ensayar una propuesta didáctica es llevándola a la luz pública, sometiéndola a la crítica y comunicándola a otros a fín de conseguir una extensión suficiente para corroborar sus ventajas e inconvenientes.

\section{NECESIDAD DE MODIFICAR LA PRÁCTI- CA EXISTENTE}

Nuestra experiencia docente en la enseñanza de la relatividad, ya fuese en el bachillerato LOGSE o durante seminarios en el marco del antiguo COU, nos había convencido de las graves insuficiencias de los esquemas didácticos al uso. Algunas de aquéllas eran:

1)Persistencia en los alumnos de la vaga sensación de que las alteraciones relativistas de longitudes y duraciones se debían a distorsiones en la percepción del observador o a efectos mecánicos sufridos por reglas y relojes debido a las elevadas velocidades de los sistemas de referencia en juego.

2) Incapacidad para captar coherentemente el origen y aplicación de la fórmula relativista de composición de velocidades.

3) Incomprensión de la presunta «masa relativista que se incrementa con la velocidad», fenómeno que a menudo se concibe por los estudiantes como una suerte de «creación de materia por arte de magia» en el seno del cuerpo móvil.

4) Incoherencias en la relación entre las ideas clásicas y relativistas sobre la energía.

5) Falta de claridad en el paso de la relatividad especial a la general y dudas sobre la pertinencia del nombre de esta última.

La insuficiencia del modelo usual quedaba con frecuencia plasmada en preguntas realizadas por los alumnos a las cuales los métodos llevados a la práctica hasta ese momento eran incapaces de responder. Algunas de tales preguntas recogen y concretan los puntos esbozados anteriormente, y enfrentan al profesor a interrogantes concretos cuya resolución en el marco de los contenidos habituales de enseñanza de la relatividad es francamente imposible. Por ejemplo:

1) Si en la relatividad especial, utilizando la «masa relativista variable con la velocidad» habla de energía cinética, ¿por qué no se introduce jamás el concepto de energía potencial relativista?

2) Si las coordenadas de posición y tiempo se transforman entre sistemas de referencia inerciales mediante las transformaciones de Lorentz, ¿cómo se transforma la presunta energía cinética entre referenciales en movimiento inercial?

3) Si la luz no tiene masa, ¿cómo puede ser afectada por la gravedad? Y si se responde que «porque tiene energía», ¿cómo puede tener energía y no masa si de acuerdo con Einstein siempre $\mathrm{E}=\mathrm{mc}^{2}$ ?

4) ¿Cómo se pasa de la relatividad especial, en la que sólo se habla de transformaciones de Lorentz, a la relatividad general, donde la idea básica es el espaciotiempo curvo?

5) ¿Qué tiene que ver la relatividad general, que debería relativizar todos los movimientos, con la gravitación? Es que no hay movimientos acelerados debidos a otras fuerzas que no sean la gravitatoria?, ¿y es que no hay ya diferencia entre movimientos acelerados y no acelerados en esta teoría?

La resolución de todas estas dudas legítimas pasa por la adopción del marco geométrico espacio-temporal verdaderamente propio de la teoría de la relatividad especial. Y ésa es la pretensión de la propuesta didáctica alternativa que a continuación se esbozará como hipótesis a debatir.

\section{UNA PROPUESTA DIDÁCTICA ALTERNA- TIVA}

Nuestro objetivo, a la hora de elaborar una nueva propuesta didáctica capaz de subsanar las carencias y malentendidos de la actual, se centraba en la transmisión clara y definida de una visión global de la teoría que permitiese al alumno experimentar un cambio epistemológico, una modificación de sus concepciones sobre el mundo físico, similar al que se produce en la transición desde el pensamiento aristotélico al paradigma científico de Galileo-Newton. Nuestra aspiración no podía ser menos ambiciosa, ya que, de lo contrario, habríamos fracasado en nuestro empeño de imbuir a los estudiantes de la cosmovisión revolucionaria inherente a la teoría de la relatividad (Berstein, 1992). Para ello era necesario articular nuestras acciones en torno a la serie de ideas básicas a asimilar por el alumno. Éstas eran: 
1) Necesidad de modificar las ideas clásicas sobre la relatividad del movimiento para extenderlas a los fenómenos electromagnéticos, lo que conduciría a la teoría de Einstein

2) Importancia fundamental en este nuevo planteamiento de conceptos tales como espacio-tiempo, suceso espacio-temporal, línea de universo o diagrama de Minkowski, ideas tratadas si acaso marginalmente en casi todos los libros de texto.

3) Entendimiento de todos los fenómenos relativistas (contracción de longitudes, dilatación de duraciones, composición subaditiva de velocidades, etc.) como efectos puramente espacio-temporales, fruto de una geometría que no es la euclídea ordinaria.

4) Renuncia a nociones extrañas a la relatividad, como masa incrementada con la velocidad, que tan solo aportan confusión a un panorama ya de por sí complejo.

\section{Contenido de la propuesta alternativa}

Al final de este artículo (Anexo I) se reproduce un resumen esquemático de los contenidos de nuestra propuesta didáctica alternativa, pero en este apartado realizaremos un breve comentario explicativo de cada uno de sus epígrafes.

Los tres primeros se dedican, a modo de introducción, a considerar el problema de la llamada crisis de la física clásica (concebida así por nosotros ahora, y no por sus coetáneos, la cual abarca no sólo el problema relativista sino también los balbuceos de la teoría cuántica e incluso los preludios de la moderna teoría del caos), las dificultades aparejadas con la relatividad galileana clásica y los enigmas surgidos a raíz de la propagación de la luz cuya velocidad parecía ser independiente de cualquier sistema de referencia. Estos tres puntos aparecen suficientemente bien tratados en las unidades didácticas típicas, por lo que no insistiremos mucho más sobre ellos. Tan sólo queremos incidir en tres aspectos: a) la crisis de la física clásica no era sentida como tal por sus contemporáneos (Sánchez Ron, 1983); b) los fenómenos electromagnéticos suponían un desafío insalvable para la relatividad galileana (Hoffmann, 1985); y c) la invariancia de dicha velocidad parecía incompatible con una relativización del electromagnetismo (Einstein, 1984).

Todas estas circunstancias condujeron al desarrollo de la teoría de la relatividad especial por parte de Einstein, como se explica en el apartado tercero, donde se desarrolla de modo casi rigurosamente histórico el contenido de su artículo de 1905. Aquí es necesario resaltar que Einstein no se vio movido a desarrollar su teoría por la exigencia de responder a los resultados negativos del experimento de Michelson-Morley (Sánchez Ron, 1983), sino por el deseo de extender el principio de relatividad a toda la física (Bunge, 1961). Buen momento para que el profesor explique a los alumnos la gran influencia ejercida por los criterios estéticos y formales de los investigadores científicos en el curso de sus indagaciones.
El punto cuarto introduce las ideas que fundamentarán la práctica posterior de esta propuesta didáctica. Siempre bajo la guía y supervisión del profesor, los estudiantes serán conducidos a deducir las principales características cualitativas de los diagramas de Minkowski (Bunge, 1967), notando que la geometría que rige en ellos es distinta de la euclídea habitual (Margenau, 1970). Ésta es una de las raíces de las peculiaridades de la física relativista, así como la inclusión del tiempo en un entramado de cuatro dimensiones denominado espaciotiempo. En estrecha analogía con el módulo de un vector en la geometría común, el tamaño de un segmento espacio-temporal (el intervalo entre dos sucesos, por ejemplo) es independiente del referencial, y son sus diferentes proyecciones sobre los ejes de espacio y de tiempo de los diversos referenciales en movimiento inercial relativo, las responsables de la relatividad de las longitudes y de las duraciones (Rucker, 1987). De este modo puede contribuirse a disipar en mayor medida las reservas de los estudiantes sobre el origen de tales efectos.

El quinto apartado se dedica a demostrar que desde este punto de vista es innecesario, e incluso abiertamente falso, el concepto de masa que se incrementa con la velocidad. La aceleración decreciente de un cuerpo acelerado -lo que le impide superar la velocidad $c$ - puede explicarse perfectamente sobre la base de la geometría de Minkowski (Baierlein, 1992) como un efecto puramente espacio-temporal (Strnad, 1991; Wangsness, 1992; Alemañ, 1997). Del mismo modo, la masa y la energía se consideran ahora magnitudes idénticas -dos nombres para un mismo concepto- que pueden referirse o bien al módulo del vector energía-impulso (energía-masa propia) o bien a su primera componente (masa-energía relativa), con lo que desaparece cualquier mención de la energía cinética o potencial (Terletskii, 1968; Alemañ, 1998).

El sexto y último apartado trata de establecer, ahora de manera coherente, un vínculo claro entre la relatividad especial y la general. Si la primera trataba con un espacio-tiempo llano, la segunda lo hace con uno curvo (Misner, Thorne y Wheeler, 1973) e incorpora en él la interacción gravitacional al precio de perder la total relativización del movimiento. Así es pese a su nombre: la relatividad general mantiene la distinción entre movimientos inerciales y acelerados, por lo que su nombre debe considerarse fruto de un malentendido histórico que por la fuerza de la tradición ha perdurado (Friedman, 1992).

\section{ESBOZO PSICOPEDAGÓGICO DEL APREN- DIZAJE}

A continuación bosquejaremos el esquema psicológico y pedagógico que consideramos más apropiado en el marco abierto de nuestra propuesta sugerida, ya que el desarrollo teórico de «cómo» se enseña es tan importante como el desarrollo teórico de «qué» se enseña. A menudo las bases del trabajo didáctico realizado no 
aparentan explicitar el plano psicológico del aprendizaje, por lo que es necesario hacer referencias suficientes sobre las concepciones personales que elaboran los alumnos y acerca de las dificultades psicoevolutivas que un tema tan complejo como éste suscita inevitablemente.

Las ideas previas de los alumnos relacionadas con este tema, y que en el presente caso pueden actuar dificultando la adquisición de nuevos conocimientos, son básicamente dos:

- En primer lugar, los estudiantes no llegan a asumir del todo correctamente el contenido esencial del principio de relatividad galileano. Parece subyacer en ellos la noción de que, a pesar de la indistinguibilidad proclamada por este principio, hay algo que privilegia unos estados como de «reposo absoluto», de forma que el desplazamiento respecto a ellos constituirá un «movimiento absoluto». El movimiento uniforme de un tren, por ejemplo, es aceptado prima facie por los alumnos como en todo equivalente al reposo de un observador sobre el andén. Pero, a poco que se indague sobre la cuestión, aflora de inmediato el convencimiento implícito en ellos de que el observador quieto en el andén está «verdaderamente en reposo»y son los del tren los que «se mueven».

- En segundo lugar, tampoco albergan, los alumnos, la menor sombra de duda sobre la validez absoluta y universal del teorema de Pitágoras y, por ende, de toda la geometría euclídea. Esta convicción supone un importante obstáculo a la hora de introducir las ideas fundamentales del espacio-tiempo de Minkowski, donde las reglas geométricas son distintas de las euclídeas. Es preciso, pues, un esfuerzo suplementario para hacerles ver que los teoremas matemáticos -de forma semejante a las teorías físicas- poseen unas condiciones de validez (verbigracia, el cumplimiento de sus axiomas) fuera de las cuales su vigencia no está en absoluto garantizada. Ejemplos como los del trazado de un triángulo sobre una superficie esférica - la de la Tierra, sin ir más lejosresultan de gran ayuda para aclarar este punto.

\section{Objetivos de aprendizaje}

Los objetivos perseguidos por nuestra propuesta didáctica pueden enumerarse como sigue:

1) Asunción por los alumnos del principio galileano de relatividad, así como de la extensión del mismo hasta el principio especial relativista de Einstein.

2) Comprensión de que las transformaciones de Lorentz entre sistemas de referencia inerciales incluyen, como caso particular, las de Galileo y que constituyen por sí mismas un vínculo nuevo y profundo entre la mecánica y el electromagnetismo.

3) Asimilación del formato geométrico introducido en la relatividad por Minkowski y del crucial de espaciotiempo relativista, algunas de cuyas consecuencias son:
4) Rechazo del equívoco concepto de masa variable con la velocidad, fruto únicamente de la interpretación de ideas relativistas desde una perspectiva newtoniana (Alemañ, 1997, 1998), junto a una adecuada comprensión de la equivalencia entre masa y energía.

5) Preparación de la base conceptual suficiente para abordar una somera introducción a los aspectos cualitativos elementales de la relatividad general.

\section{Estrategia didáctica}

La estrategia empleada se plasmó en la siguiente secuencia de actuaciones:

a) Planteamiento de las insuficiencias de la física clásica a la luz de las nuevas evidencias empíricas y de sus propias limitaciones internas como teoría total de la naturaleza. Esta situación exige ser superada mediante una nueva teoría del mundo físico, que en este caso es la relatividad

b) Comparación con la física clásica a la hora de ensayar un formato geométrico para la teoría, en donde los alumnos han de recurrir a la costumbre-generalmente adquirida en el nivel preuniversitario- de representar desplazamientos, velocidades y aceleraciones geométricamente en el ámbito de la cinemática newtoniana. Éste es el momento para exponer que, al igual que la física clásica se basaba en una geometría euclídea del espacio y del tiempo, la teoría de la relatividad, como nueva teoría que es, probablemente precise de una nueva geometría. Y, en efecto, así es, como se debe desprender de la presentación en este punto de la geometría de Minkowski.

c) Construcción del entramado espacio-temporal relativista por comparación y contraste con los diagramas y gráficos similares en la física clásica.

d) Introducción de la idea de que todos los fenómenos en la relatividad especial pueden ser interpretados fructíferamente en el seno de esta perspectiva espacio -temporal.

e) Confrontación constante de los nuevos contenidos con los prejuicios del alumno heredados de la física newtoniana (e incluso, a veces, de la aristotélica) y de la geometría euclídea.

f) Insistencia constante en el hecho de que la nueva teoría, la relatividad (tanto en su versión especial como en la general), nace del uso del mismo método científico -experimental y lógico-deductivo- que dio origen a las teorías de la física clásica, de las que tan seguros nos sentimos y que tan intuitivas consideramos.

\section{Etapa de evaluación}

En la etapa evaluadora del proceso de aprendizaje, extendida a lo largo de todo el desarrollo de la unidad didáctica, podemos distinguir, a su vez, tres fases: 
1) Inicial: Esta primera fase se sustancia a través de la discusión de ideas previas (significado de la relatividad del movimiento, la velocidad de la luz, la validez de la geometría euclídea, etc.) de los alumnos. El trabajo en grupo y la puesta en común de estas ideas constituyen el eje fundamental que nos permitirá avanzar hacia la segunda fase.

2) Cambio conceptual: En esta fase se produce el cambio conceptual propiamente dicho gracias al contraste de las ideas previas, ya elucidadas en la fase anterior, con el resultado de ejercicios individualmente realizados en momentos seleccionados, con razonamiento del propio alumno inducidos por el profesor y con los datos aportados como información nueva por artículos, textos escogidos o por el mismo docente.

3) Etapa de refuerzo: Por último se pretende asentar el conocimiento adquirido mediante la realización de ejercicios de refuerzo sobre los aspectos clave del tema tratado. En todas estas fases se produce simultáneamente una evaluación de otros aspectos relevantes del aprendizaje, como son la motivación del alumno, el interés despertado en él mismo y su capacidad de autoevaluación. Las actividades a realizar para ello son diversas: seguimiento de los cuadernos personales de trabajo, reflexión colectiva sobre las dificultades halladas en el tema, discusión sobre el contraste entre el tiempo previsto para el desarrollo de la unidad y el tiempo real empleado, etc.

\section{CONCLUSIONES}

Pese a la incipiencia del proceso que toda nueva propuesta didáctica implica, podemos establecer de manera provisional las siguientes conclusiones a la espera del enriquecimiento en nuevas perspectivas pedagógicas que propicia todo debate epistemológico abierto.

- Parece posible construir un proyecto didáctico alternativo en el tema de relatividad fundado en una perspectiva espacio-temporal de dicha teoría, lo que nos acerca mucho más al verdadero contenido de la misma que las unidades didácticas más usuales, pese a las dificultades surgidas al conectar las representaciones visuales y gráficas relativistas con las concepciones personales de los alumnos sobre las propiedades del espacio y el tiempo en el marco de su visión intuitiva del mundo.

- Lejos de todo dogmatismo, somos conscientes de que esta propuesta presenta ventajas e inconvenientes. Las ventajas son, entre otras, una mayor comprensión y capacidad de representación visual y gráfica, por parte del alumno, de los efectos relativistas. Los inconvenientes, por otro lado, estriban en la necesidad de habituarse a una geometría que no es la euclídea ordinaria. Sin embargo, una vez admitidas las reglas de la geometría de Minkowski, los efectos relativistas aparecen como resultados lógicos y naturales de las mismas (Feigenbaum, 1990).

- Entendemos que existe el peligro de abocar a los alumnos a un cambio conceptual incompleto y, por ello, falso. Así ocurriría si se les indujese a interiorizar la idea de que el diagrama de Minkowski «va creciendo o desplegándose conforme pasa el tiempo», error este que se detecta incluso en algunos textos especializados (Sazànov, 1990). Otros posibles errores serían la suposición de que la energía adquirida por un cuerpo, al ser acelerado, «se invierte, en parte, en variar su velocidad $\mathrm{y}$, en parte, en variar su masa» (error ligado al malentendido de la «masa variable con la velocidad») o la idea de que masa y energía son meramente dos conceptos dispares relacionados por una constante universal $c$. Es necesario, para evitarlo, destacar muy claramente los aspectos conceptuales básicos de la teoría confrontándolos cuantas veces sea preciso con los prejuicios de los propios alumnos y con las vulgarizaciones erróneas de la misma.

- Si bien los resultados numéricos son casi siempre idénticos, el punto de vista conceptual es radicalmente distinto entre el planteamiento espacio-temporal y el tradicional. Por ese motivo proponemos la modificación de los contenidos de los temarios oficiales así como de las pruebas de acceso a la universidad con el fin de eliminar, de los mismos, términos proscritos en una enseñanza rigurosa de la relatividad, como es el caso, por ejemplo, de la desafortunada masa variable con la velocidad, popularizada por algunos de los autores de los materiales curriculares actuales (Gil, Senet y Solbes, 1988; Domènech, 1998).

- El procedimiento más aconsejable para abordar este ensayo parece que es la detección de las ideas previas del alumno (con la correspondiente reflexión colectiva y autocrítica), la modificación de dichas ideas mediante aporte dirigido por el profesor de nueva información (datos experimentales, observaciones) y, finalmente, el refuerzo de los conocimientos adquiridos por medio de ejemplos numéricos sencillos. En todas estas etapas es preciso destacar la importancia del contexto teórico en la interpretación de las fórmulas y de los conceptos subyacentes. 


\section{REFERENCIAS BIBLIOGRÁFICAS}

ALEMAÑ, R. (1997). Errores comunes sobre relatividad entre los profesores de enseñanza secundaria. Enseñanza de las Ciencias, 15(3), pp. 301-307.

ALEMAÑ, R. (1998). Grandes metáforas de la física. Madrid: Celeste Ediciones.

BAIERLEIN, R. (1992). Newton to Einstein. The trail of light. Cambridge: Cambridge University Press.

BERSTEIN, J. (1992). Einstein, el hombre y su obra. Madrid: MacGraw-Hill.

BUNGE, M. (1961). Laws of physical laws. American Journal of Physics, 29, pp. 518-522.

BUNGE, M. (1967), Foundations of Physics. Nueva York: Springer Verlag.

CALVO, A. y SEGUÍ, J. (1996). La enseñanza de la relatividad en los nuevos programas de física de LOGSE. Revista Española de Física, 10(3), pp. 44-49.

DOMÈNECH, A. (1998). El debate sobre la masa relativista: el problema definicional y otros aspectos epistemológicos. Enseñanza de las Ciencias, 16(2), pp. 331-339.

EINSTEIN, A. (1984). Sobre la teoría de la relatividad especial y general. Madrid: Alianza.

FEIGENBAUM, M.J. (1990). $E=m c^{2}$. Cambridge: Cambridge University Press.

FRIEDMAN, M. (1992). Fundamentos de las teorías del espaciotiempo. Madrid: Alianza.

GIL, D., SENENT, F. y SOLBES, J. (1988). E = mc ${ }^{2}$, la ecuación más famosa de la física: una incomprendida. Revista Española de Física, 2, pp. 53-55.
HOFFMANN,B. (1985). La relatividady sus orígenes. Barcelona: Labor.

MARGENAU, H. (1970). La naturaleza de la realidad física. Madrid: Tecnos.

MEC. RD 1179/1992 del 2 de octubre por el que se establece el currículo de bachillerato (BOE del 21).

MISNER, C., THORNE, K. y WHEELER, J. (1973). Gravitation. San Francisco: Freeman.

PENROSE, R.(1991). Lanueva mente del emperador. Barcelona: Mondadori.

RUCKER, R. (1987). La cuarta dimensión. Barcelona: Salvat.

SÁNCHEZ RON, J.M. (1983). Origen y desarrollo de la relatividad. Madrid: Alianza.

SAZÀNOV, A. (1990). El universo tetradimensional de Minkowski. Moscú: MIR.

SOLBES, M.J. (1993). Física. Materiales curriculares del $M E C$. Madrid: MEC.

SCHWINGER, J. (1995). El legado de Einstein. Barcelona. Investigación y Ciencia.

STRNAD, J. (1991). Velocity-dependent mass or proper time? Eur. J. Phys., 12, pp. 69-73.

TERLETSKII, Y.(1968). Paradoxes in the Theory of Relativity. Nueva York: Plenum Press.

WANGSNESS, R. (1992). Campos electromagnéticos. México: Limusa.

[Artículo recibido en mayo de 1999 y aceptado en octubre de 1999.] 


\section{ANEXO I \\ ESQUEMA DE LA UNIDAD DIDÁCTICA ALTERNATIVA}

Llevaremos a cabo la esquematización de nuestra unidad didáctica alternativa sobre el tema de relatividad dividiendo la secuenciación del proceso de aprendizaje en tres etapas: inicición (tratamiento de la situación de partida), innovación (adquisición de nuevos conocimientos), aplicación (utilización de los nuevos contenidos en aplicaciones diversas) y revisión (recapitulación y refuerzo del aprendizaje). En cada una de ellas enumeraremos las cuestiones básicas que darán paso a la discusión en el grupo y en torno a las cuales se elaborarán las actividades de enseñanza-aprendizaje.

1. Iniciación. En esta etapa se pretende explicitar el dilema al que había llegado la física decimonónica en relación con la relatividad del movimiento y los fenómenos electromagnéticos, los cuales no parecían encajar en aquélla. Se destaca la gran cantidad de trabajos experimentales al respecto (entre ellos, pero ni mucho menos el único, el de Michelson) y la curiosa influencia que ejercieron sobre Einstein. Los alumnos, con el uso de todo el material bibliográfico asequible, deben aprender a contextualizar los avances científicos, exponiendo sus ideas en pequeños grupos y poniendo en común el contenido de una hoja de trabajo que se irá completando a lo largo de las sucesivas etapas en el desarrollo del tema. Las cuestiones centrales a discutir serían:

- ¿Qué problemas encontró la física clásica al final del siglo XIx? ¿Cómo se relacionaban con otras ramas de la física?

- ¿Qué sucedía con la relatividad del movimiento en relación con las ondas electromagnéticas? ¿Qué consecuencias tenía la constancia de la velocidad de la luz?

- ¿Qué experimentos se llevaron a cabo al respecto? ¿Qué explicaciones se dieron al experimento de Michelson-Morley?

- ¿Influyó este experimento en Einstein? ¿Por qué suele afirmarse que sí?

2. Innovación. Introducimos ahora nuevos conceptos, ya puramente relativistas la mayoría de ellos, siguiendo la línea del artículo de Einstein de 1905, cuyo prólogo -donde el sabio alemán explica las razones que le han llevado a su teoría- se lee en clase. Los alumnos deben asumir ahora la ampliación del principio de relatividad especial a toda la física expresado en las transformaciones de Lorentz. Estas transformaciones sugieren el cambio del espacio y tiempo clásicos por un nuevo marco tetradimensional, el espacio-tiempo, donde las reglas geométricas difieren de las euclídeas. Las consecuencias físicas se tratan posteriormente.

\subsection{Principio especial de relatividad}

- ¿Cómo resuelve Einstein la aparente contradicción entre la relatividad del movimiento y la constancia de $c$ ?

- ¿Por qué se dice que el tiempo es relativo? ¿Qué diferencias encuentras con el tiempo newtoniano?

2.2. Consecuencias del principio (contracción de longitudes, dilatación de períodos, composición de velocidades).

- ¿Qué transformaciones relacionan ahora los sistemas inerciales?

- ¿Cómo deben interpretarse las contracciones de longitudes y dilataciones de tiempos?

- ¿Qué ocurre con la suma clásica de velocidades?

- ¿Pueden mantenerse leyes físicas que permitan $v>c$ ?

2.3. Modelo geométrico: espacio-tiempo de Minkowski

- ¿Qué se entiende en relatividad por «espacio-tiempo»?

- ¿Cómo se expresa geométricamente la relatividad especial en el espacio-tiempo?

- ¿Qué ventajas posee este planteamiento?

\subsection{Masa y energía}

- ¿Varía la masa con la velocidad? ¿Por qué aparece así en los libros de texto?

- ¿Qué significa realmente la ecuación $\mathrm{E}=\mathrm{mc}^{2}$ ?

3. Aplicaciones. Se trata aquí de que el alumno trate de concebir, en la medida de lo posible, las repercusiones que sobre las distintas ramas de la ciencia, la tecnología y la sociedad han tenido las teorías de Einstein. 


\section{INNOVACIONES DIDÁCTICAS}

- Realícese un trabajo en pequeños grupos - para la posterior puesta en común en el aula- sobre la influencia ejercida por la relatividad sobre la astrofísica, la cosmología, la física de altas energías, los aceleradores de partículas, etc.

- ¿Cómo se entiende desde el punto de vista de Einstein la gravedad?

- Nómbrense aplicaciones y consecuencias tecnológicas y sociales de la relatividad.

4. Revisión.

- ¿Cómo podríamos realizar un esquema de las ideas aprendidas?

- ¿Qué debemos modificar en los conceptos discutidos al elucidar las ideas previas?

- ¿Dónde se encuentran las mayores dificultades de la unidad didáctica?

\section{ANEXO II \\ CUESTIONARIO FINAL DE AUTOEVALUACIÓN}

Señálese la contestación considerada más correcta a la pregunta de cada apartado. En caso de que sea elegida la opción $c$ ), «Otras respuestas», expónganse brevemente en lugar aparte los argumentos que se crean oportunos.

1. ¿A qué se debió la llamada «crisis de la física del siglo XIX»?

a) Al conflicto entre el movimiento absoluto y la relatividad del movimiento.

b) A la extraña interacción de la radiación con la materia.

c) A los dos anteriores.

d) Otras respuestas.

2. ¿Cómo influyó el experimento de Michelson-Morley sobre el pensamiento de Einstein?

a) En nada.

b) Fue la causa que le animó a elaborar su teoría de la relatividad especial.

c) Este experimento fue una confirmación posterior de la relatividad.

d) Otras respuestas.

3. ¿Qué movimientos estudia la teoría especial de la relatividad?

a) El movimiento arbitrario de un punto material respecto a algún otro de referencia.

b) Movimientos arbitrarios respecto a referenciales en movimiento inercial.

c) Toda clase de movimientos.

d) Otras respuestas.

4. Los fenómenos relativistas de dilatación de las duraciones y contracción de las longitudes:

a) Se deben al efecto de la velocidad sobre los átomos de reglas y relojes de medida.

b) Son efectos aparentes provocados por velocidades cercanas a la de la luz.

c) Son efectos espacio-temporales físicamente reales y objetivos.

d) Otras respuestas. 


\section{INNOVACIONES DIDÁCTICAS}

5. ¿Por qué la velocidad de la luz es un límite físico insuperable?

a) Es tan sólo un problema tecnológico.

b) Porque la masa del cuerpo se incrementa conforme su velocidad se aproxima a $c$.

c) Por un efecto relativista combinado del espacio y el tiempo.

d) Otras respuestas.

6. ¿Qué es el «espacio-tiempo»?

a) Una invención errónea de la literatura de ciencia-ficción.

b) Una invención matemática y puramente abstracta de los físicos.

c) Un concepto objetivo que sustituye el espacio y el tiempo por separado.

d) Otras respuestas.

7. ¿De qué se ocupa la relatividad general?

a) Incluye las radiaciones y la física cuántica en la relatividad.

b) Relativiza todo movimiento, inercial o no.

c) Construye una teoría relativista de la gravitación.

d) Otras respuestas.

\section{ANEXO III \\ PRUEBA DE EVALUACIÓN SOBRE RELATIVIDAD (DOS HORAS)}

1. La vida media de un muón de los rayos cósmicos es de 6,5 microsegundos (6,5 (s) cuando está en reposo respecto a nosotros. $\mathrm{Si}$ su velocidad relativa al suelo es de $v=0,994 c$, estima la longitud de la distancia que recorren los muones, considerada en su sistema de referencia, antes de desintegrarse

2. Una nave espacial transporta desde la Tierra al planeta $X$ un sabroso cargamento de bizcochos cuya duración tras el envasado, en tiempo de la Tierra, es de seis meses. Si la distancia desde nuestro mundo al planeta X es de 10 años-luz, ¿a qué velocidad debe moverse el carguero espacial para llegar al planeta $\mathrm{X}$ antes de que los bizcochos caduquen?

3. Una astronave pasa junto a la Tierra a una velocidad de $0,5 c$, justo cuando uno de sus tripulantes dispara una pistola de electrones en el mismo sentido de avance de su nave. Si los electrones se mueven a $0,85 \mathrm{c}$ respecto al cosmonauta, ¿cuál será su velocidad respecto a un observador en la Tierra?

4. En un acelerador de partículas, un protón adquiere una energía respecto a nosotros que es un $25 \%$ superior a su energía propia. Sabiendo que su masa propia es $\mathrm{m}=1,6725\left(10^{(27} \mathrm{k}\right.$, calcula: $\left.a\right)$ su energía propia, $\left.b\right)$ su energía relativa a nosotros, y $\left.c\right)$ su velocidad relativa a nosotros.

5. Pregunta suplementaria opcional. Un colegio tiene dos aulas situadas una en la planta baja y otra en otro piso del edificio. Cada una de ellas tiene su propio sistema de relojes y timbres para señalar la hora de salida. De acuerdo con los principios de la relatividad general, ¿cuál de las dos aulas convendría escoger a los alumnos que deseasen salir antes?

\section{NOTA}

Realiza todos los esquemas y diagramas de Minkowski que desees para aclarar el significado de los problemas y sus respuestas. 